Ann. Zootech., I97I, 20 (4), 497-507.

\title{
INFLUENCE D'UNE INJECTION DE SULFATE DE MAGNÉSIUM SUR LE DÉROULEMENT DE LA GLYCOGÉNOLYSE POST MORTEM DANS LE MUSCLE DE PORC
}

\author{
G. MONIN \\ avec la collaboration technique de Colette VIGNE \\ Station de Recherches sur la Viande, \\ Centre de Recherches de Clermont-Ferrand, I. N. R. A., \\ 63 - Saint-Genès-Champanelle
}

\section{RÉSUMÉ}

L'influence de l'injection de sulfate de magnésium, immédiatement avant l'abattage, sur le déroulement de la glycogénolyse musculaire post mortem a été étudiée sur sept porcs de race Large White et 5 porcs de race Piétrain comparés à un nombre égal de témoins de chaque race.

Ce traitement a pour effet de supprimer la transmission aux muscles des violentes stimulations nerveuses engendrées par les opérations d'abattage; il entraîne un ralentissement de la chute du $\mathrm{pH}$ et de la glycogénolyse post mortem au niveau du muscle Longissimus dorsi et surtout du muscle Rectus abdominis, plus riche en fibres rouges. Dans le Longissimus dorsi cet effet de l'injection d'ions $\mathrm{Mg}^{++}$est beaucoup plus net chez les porcs de race Large White que chez les Piétrain. Les relations entre l'action du magnésium et certaines caractéristiques physiologiques du muscle, telles que l'irrigation sanguine et le type métabolique des fibres, sont discutées.

Le traitement entraîne une amélioration de la qualité de la viande, surtout du pouvoir de rétention d'eau. Néanmoins cet effet reste très insuffisant pour assurer l'obtention d'une viande de qualité satisfaisante, principalement dans la race Piétrain.

\section{IN'TRODUC'TION}

Il arrive fréquemment, dans l'espèce porcine, que la glycogénolyse musculaire post mortem se déroule de façon anormalement rapide. Si les conséquences néfastes de ce phénomène sur certaines caractéristiques de qualité des viandes, telles que la coloration et le pouvoir de rétention d'eau, sont maintenant bien établies (BRISKEY 
et WISMER-PEDERSEN, I96I) nos connaissances sur son origine et les moyens d'y remédier sont encore très incomplètes. On sait que les causes en sont d'ordre physiologique : 1'irrigation sanguine déficiente et l'anoxie tissulaire jouent un rôle déterminant (LUDVIGSEN, I957 ; BUGARD et al., I963) ; BENDALI. (I965) a montré que la suppression totale, par curarisation, des contractions musculaires au moment de l'abattage entraîne un net ralentissement de la chute du $\mathrm{pH}$ musculaire post mortem. Toutefois les observations de LISTER (I968), LISTER et al. (I970), SAIR et al. (I970) révèlent que l'importance respective de l'anoxie et des stimulations nerveuses dans le déclenchement de l'anomalie varie largement d'une race à l'autre. C'est pourquoi il nous a paru intéressant de déterminer si la suppression des stimulations nerveuses et des contractions musculaires au moment de la mise à mort avait des répercussions identiques sur le déroulement de la glycogénolyse musculaire post mortem chez les races Large White et Piétrain. En outre, puisque l'état d'oxygénation et d'irrigation sanguine du tissu musculaire influencent la glycogénolyse, nous avons considéré deux muscles de type métabolique sensiblement différent, le Longissimus dorsi et le Rectus abdominis : chez le Porc le premier contient 20 à $25 \mathrm{p}$. Ioo de fibres rouges, le second environ 40 p. IOO (BEECHER et al., I965).

Pour réaliser la suppression des contractions musculaires au moment de l'abattage, nous avons employé le sulfate de magnésium utilisé avec succès par SAIR et al. (I970) : les ions $\mathrm{Mg}^{++}$, en inhibant la libération d'acétylcholine par les terminaisons nerveuses au niveau de la plaque motrice, s'opposent à la transmission de l'influx nerveux au niveau de la jonction neuromusculaire, mettant ainsi le muscle hors d'atteinte des stimulations consécutives aux opérations d'abattage.

\section{MATÉRIEL ET MÉTHODES (1)}

Le matériel animal utilisé lors de cette étude comprend $\mathrm{I}_{4}$ porcs de race Large White de IOO \pm to $\mathrm{kgs}$ et Io porcs Piétrain de $95 \pm 5 \mathrm{~kg}$.

Tous les animaux sont soumis à un jeûne de 24 heures. Puis dans chaque race, la moitié des porcs reçoit, par injection intraveincuse à l'oreille, $15 \mathrm{~g}$ de $\mathrm{MgSO}_{4}$ en solution à $25 \mathrm{p}$. Ioo (dose nécessaire pour provoquer une narcose complète). Deux à trois minutes après la fin de l'injection les animaux sont abattus. L'autre moitié ne reçoit aucun traitement avant l'abattage.

Dans tous les cas l'abattage comprend l'application d'un courant d'électronarcose à l'aide d'un appareil ETIM polyvalent ( $50 \mathrm{~Hz}, 200 \mathrm{~V}$, durée 5 secondes) avant la saignée.

Immédiatement après la fin de la saignée le $\mathrm{pH}$ est mesuré et des échantillons prélevés sur le muscle Longissimus dorsi, dans la région dorso-lombaire. Les mêmes opérations sont répétées trente minutes plus tard sur le muscle Rectus abdominis, puis sur les deux muscles simultanément une, deux et trois heures post mortem.

Les échantillons prélevés sont divisés en deux parties : l'une destinée au dosage du glycogène, l'autre à la détermination de l'ATP, de la phosphocréatine et de l'acide lactique. Le glycogène est dosé selon la méthode de Good, KRamer et Somogyi ( 1963 ) modifiée comme indiqué par CharPENTIER (I968) en utilisant pour le dosage du glucose final la méthode enzymatique de HuGUETT et NIXON (I957). L'acide lactique et 1'ATP sont déterminés par les méthodes enzymatiques classiques rapportées par BERGMEYER (1965) après extraction dans l'acide perchlorique 0,6 N et neutralisation de l'extrait par la potasse $5 \mathrm{~N}$. Sur ce même extrait, la phosphocréatine était déterminée par dosage de l'ATP obtenu en réalisant la réaction $\mathrm{PC}+\mathrm{ADP} \rightarrow \mathrm{ATP}+$ créatine en présence de créatine-kinase.

(1) Abréviations employées: ATP $=$ adénosine triphosphate

$\mathrm{PC}=$ Phosphocréatine 
Trois heures après l'abattage les carcasses sont placées à $+4^{\circ} \mathrm{C}$. Après 24 heures de ressuyage le jambon est détaché d'une demi-carcasse et le $\mathrm{pH}$ mesuré dans les muscles Longissimus dorsi, Biceps femoris, et Gluteus medius. Afin d'apprécier le pouvoir de rétention d'eau, le temps nécessaire au changement de coloration d'un fragment de $\mathrm{I} \mathrm{cm}^{2}$ de papier indicateur de $\mathrm{pH}$ (Prolabo universel) posé sur la coupe fraîche des muscles Longissimus dorsi, Biceps femoris et Gluteus medius est également déterminé ; ce temps étant parfois très long sur les échantillons de bonne qualité la mesure est arrêtée après trois minutes et une note est attribuée à. l'échantillon d'après l'échelle suivante :

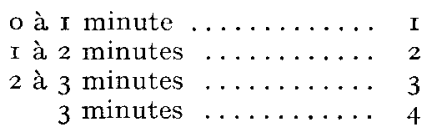

Enfin une note subjective est attribuée à chaque jambon après appréciation de sa qualité d'après son aspect extérieur (couleur, humidité, tenue) :

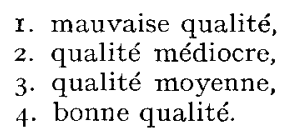

\section{RÉSULTATS}

\section{I. - Comportement des animaux pendant l'abattage}

Au cours de l'injection de sulfate de magnésium, on observe chez les animaux traités une brève phase d'excitation, précédant de peu la chute sur le sol. On peut alors constater l'absence de réaction à la douleur, ainsi qu'un relâchement musculaire dénoté par la souplesse des membres. Lors de l'application du courant d'électronarcose et de la saignée, les pores ne montrent aucune réaction. Ce comportement contraste avec les mouvements violents qui accompagnent le passage du courant chez les animaux témoins et se poursuivent durant toute la saignée.

\section{2. - Evolution du $p H$ et des divers substrats et cofacteurs de la glycogénolyse}

\section{Dans le muscle Longissimus dorsi (tabl. I).}

Chez les animaux témoins la chute de $\mathrm{pH}$ présente une grande variabilité. Si les valeurs initiales du $\mathrm{pH}$ sont voisines, elles diminuent beaucoup plus rapidement chez les animaux de race Piétrain, puisque le $\mathrm{pH}$ ultime est pratiquement atteint une heure post mortem. (Il l'est effectivement chez quatre animaux sur cinq.) L'injection de sulfate de magnésium ante mortem a pour effet d'élever notablement le $\mathrm{pH}$ initial, et ultérieurement de ralentir son évolution ; toutefois chez les porcs Piétrain la vitesse de chute du $\mathrm{pH}$ reste supérieure à celle que l'on observe chez les Large White témoins.

En ce qui concerne le taux initial et l'évolution du glycogène dans les divers groupes expérimentaux, on remarque une influence à la fois du traitement et de la race : chez les animaux traités, ce composé est présent en quantité supérieure immédiatement après la mort, et sa dégradation ultérieure est plus lente, ceci dans les deux races; mais, pour un même traitement, le taux initial de glycogène est plus élevé et sa disparition plus rapide chez les Piétrain que chez les Large White. 


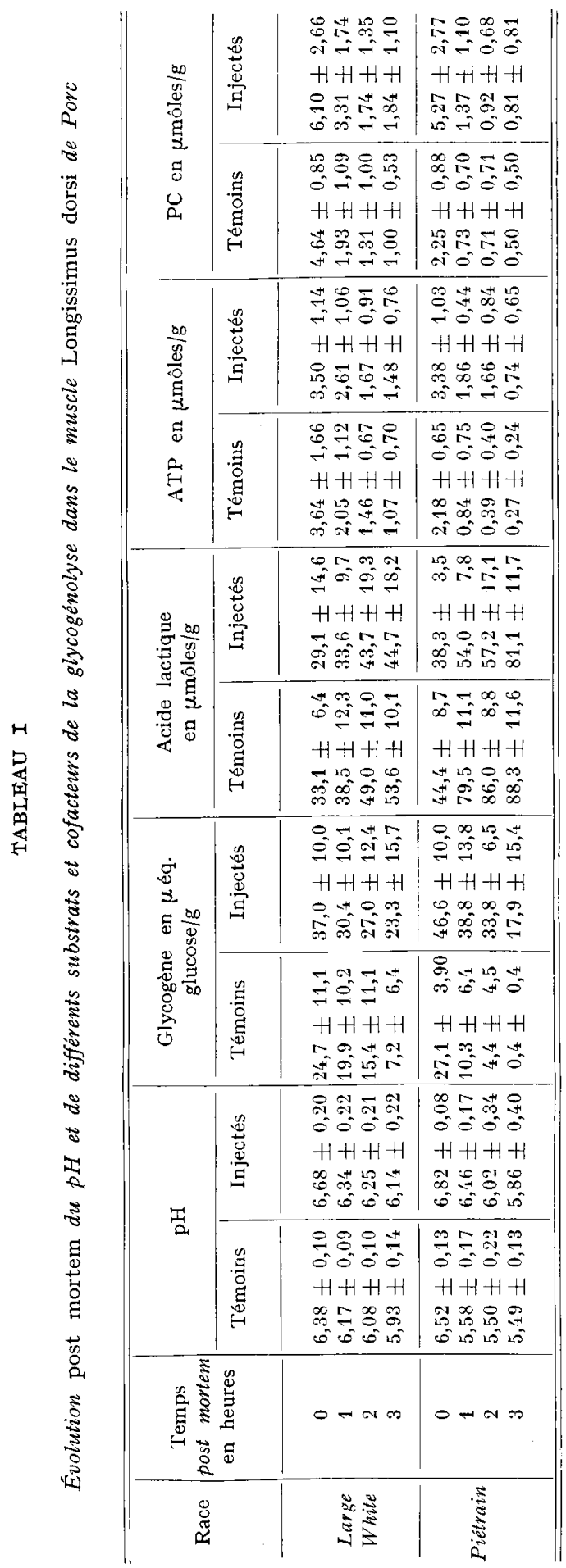




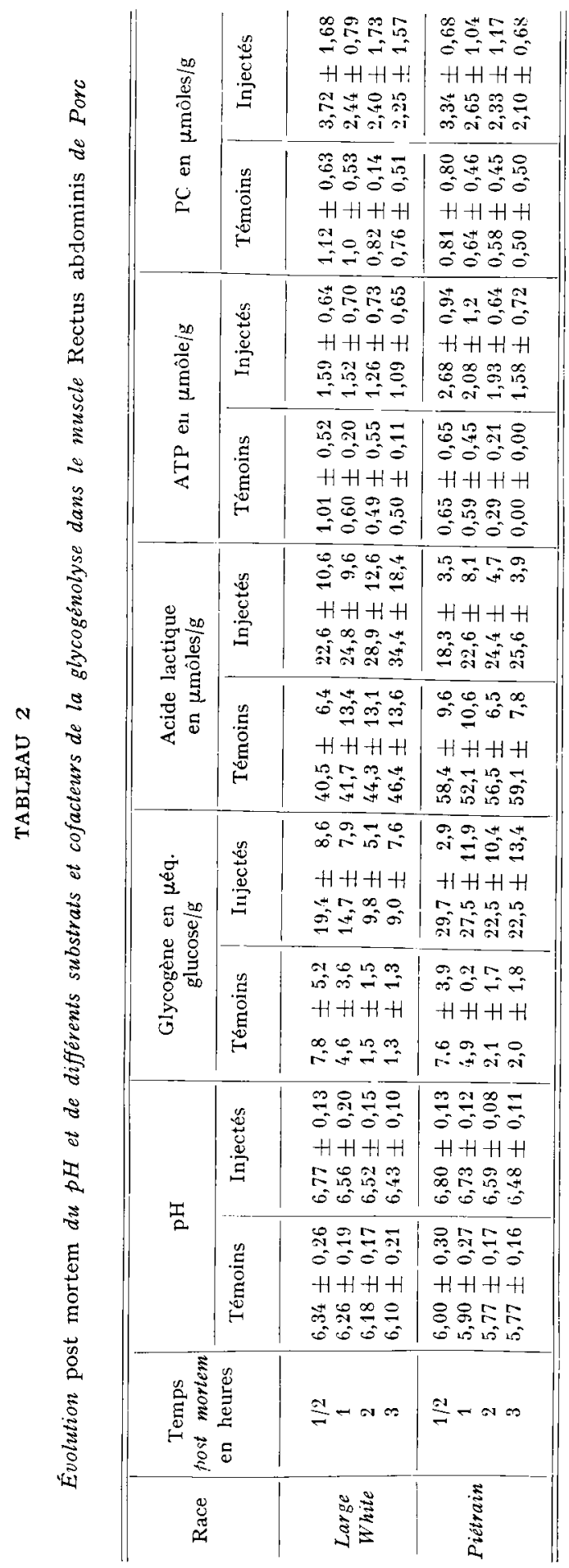


L'acide lactique est présent immédiatement après l'abattage à des taux très comparables dans les différents groupes. Son accumulation au cours des heures succédant la mort présente les mêmes caractéristiques que la dégradation du glycogène : elle est plus rapide chez les Piétrain et surtout chez les animaux témoins de cette race où le taux ultime est presque atteint I heure post mortem.

L'injection du sulfate de magnésium entraîne une augmentation notable des taux des composés phosphorylés riches en énergie (ATP et PC) : chez les Piétrain, en particulier, la teneur initiale en phosphocréatine du muscle Longissimus dorsi est plus que doublée par ce traitement $(5,3 \mu$ moles/g chez les porcs traités contre $2,3 \mu$ moles/g chez les témoins). Son influence est beaucoup moins nette chez les Large White. Il est remarquable que la variabilité du taux de phosphocréatine soit beaucoup plus grande chez les animaux injectés que chez les témoins et ceci dans les deux races (en particulier on observe chez les Large White ayant reçu des ions $\mathrm{Mg}^{++}$des taux extrêmes de 4,59 et 9,25 $\mu$ moles/g de phosphocréatine). En ce qui concerne 1'ATP les taux initiaux sont comparables dans les différents groupes $(3, \mathrm{I}$ à $3,6 \mu$ moles/g) sauf chez les Piétrain témoins où ils sont un peu plus faibles $(2,2 \mu$ moles/g). La dégradation des composés phosphorylés est particulièrement rapide chez ces derniers puisque une heure post mortem quatre animaux sur cinq présentent des taux d'A'TP et de phosphocréatine inférieurs à $0,5 \mu \mathrm{mole} / \mathrm{g}$. Ce sont les Large White injectés qui manifestent la vitesse de dégradation la plus faible à la fois pour la phosphocréatine et 1'ATP.

\section{Dans le muscle Rectus abdominis (tabl. 2).}

L'évolution du $\mathrm{pH}$ au cours des heures suivant 1'abattage présente des variations plus importantes encore dans le Rectus abdominis que dans le Longissimus dorsi. En effet, si chez tous les animaux traités la diminution du $\mathrm{pH}$ est très lente (o,I unité $\mathrm{pH} /$ heure pendant les trois premières heures), elle est extrêmement rapide chez les Piétrain témoins puisque une heure post mortem le $\mathrm{pH}$ ultime est atteint chez trois animaux sur cinq. Il est à noter que l'injection du sulfate de magnésium ante mortem ramène la vitesse d'évolution $\mathrm{d} u \mathrm{pH}$ à des valeurs comparables dans les deux races, ce qui n'était pas le cas au niveau du Longissimus dorsi.

Comme dans le Longissimus dorsi, le taux de glycogène est plus élevé dans la race Piétrain que dans la race Large White. Ceci est net chez les animaux traités; chez les témoins, on observe $30 \mathrm{mn}$ post mortem des taux identiques. Or chez les Piétrain la glycogénolyse est très avancée, comme en témoignent le $\mathrm{pH}$ et le taux d'acide lactique, ce qui laisse supposer un taux initial nettement supérieur. Tout comme celle du $\mathrm{pH}$, l'évolution des quantités de glycogène présentes dans le muscle à l'abattage est très différente selon les groupes expérimentaux, et semble la plus rapide chez les Piétrain témoins. L'injection des ions $\mathrm{Mg}^{++}$permet le maintien d'un taux de glycogène élevé $30 \mathrm{mn}$ post mortem, la différence "initiale " ainsi réalisée entre porcs témoins et traités se maintenant au cours des trois premières heures.

$\mathrm{Au}$ moment du premier prélèvement les quantités d'acide lactique présentes dans le muscle sont très variables. Très importantes chez les témoins des deux races, elles sont faibles après l'injection du sel de magnésium. Comme elles évoluent lentement dans tous les cas les valeurs observées après trois heures sont également très différentes. La variabilité des taux d'acide lactique est particulièrement élevée chez les Large White, où elle augmente en même temps que les valeurs moyennes : ceci 


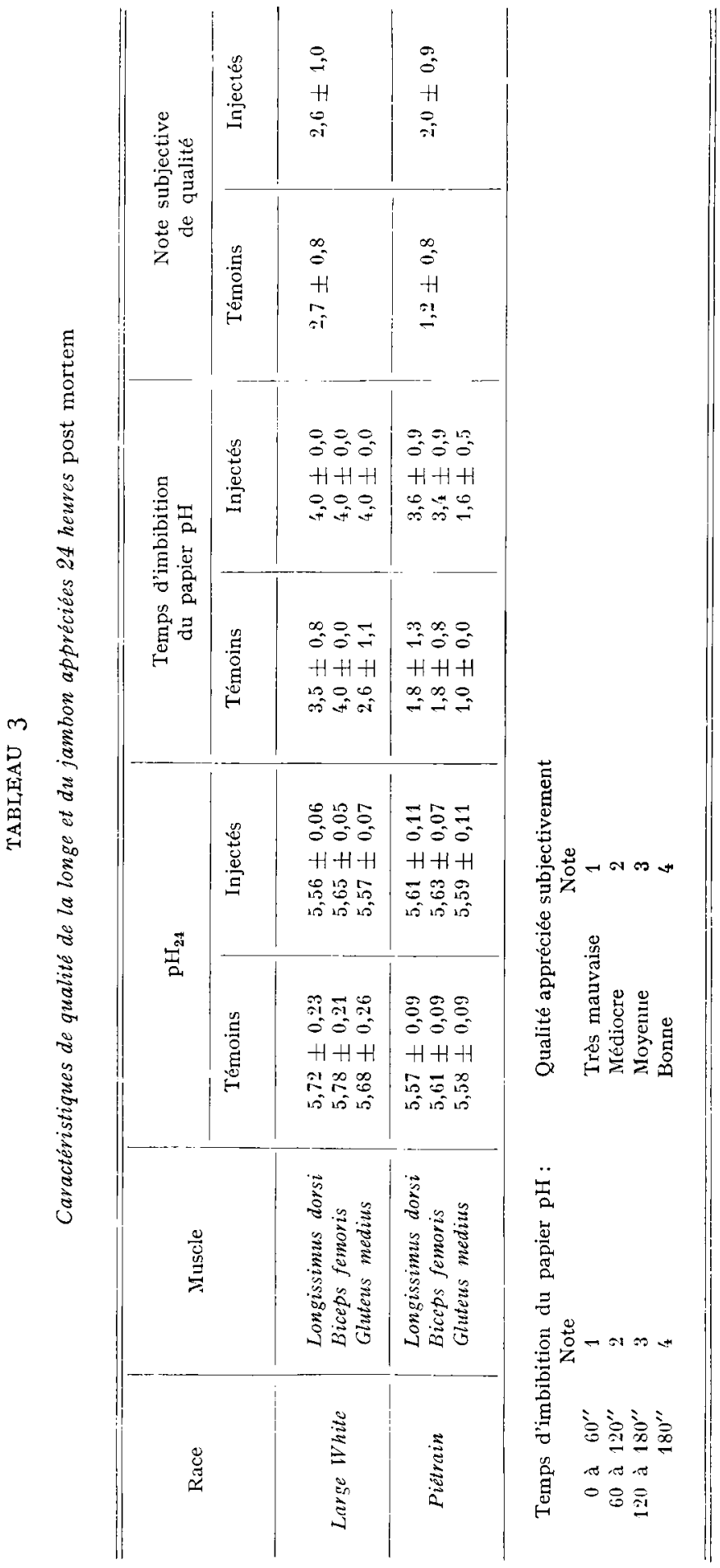


traduit des différences individuelles dans la vitesse d'accumulation de 1'acide, qui sont surtout remarquables chez les animaux traités.

Ici encore l'évolution de la phosphocréatine et de 1'ATP dans les divers groupes expérimentaux reflète la vitesse de glycogénolyse post mortem telle qu'elle est indiquée par la chute de $\mathrm{pH}$. Les animaux traités se caractérisent, par rapport aux témoins, par des taux initiaux plus élevés et une déplétion plus lente de ces detux composés phosphorylés. Ces derniers sont dégradés très rapidement chez les Piétrain témoins où l'on constate la disparition totale de l'ATP trois heures post mortem.

\section{3. - Caractéristiques de qualité de la viande (tabl. 3)}

Il y a peu de différences entre les divers groupes de porcs en ce qui concerne le $\mathrm{pH}_{24}$ dans les différents muscles observés. Il faut toutefois noter la légère diminution du $\mathrm{pH}_{24}$ chez les animaux de race Large White ayant reçu l'injection de magnésium avant l'abattage, la différence entre les deux groupes est comprise entre $0, \mathrm{I}$ I unités $\mathrm{pH}$ pour le Gluteus medius et o,I6 unités $\mathrm{pH}$ pour le Longissimus dorsi.

Par contre, la mesure du temps d'imbibition du papier $\mathrm{pH}$ posé sur la coupe fraîche des muscles met en évidence des différences assez nettes de pouvoir de rétention d'eau entre les différents muscles d'une part et les groupes expérimentaux d'autre part. Quels que soient la race et le traitement subi, le Gluteus medius possède le pouvoir de rétention d'eau le plus faible ; le Longissimus dorsi et le Biceps femoris se caractérisent par des temps d'imbibition sensiblement équivalents. L'injection de sulfate de magnésium améliore la rétention de l'eau dans les deux races ; toutefois cette caractéristique reste inférieure chez tous les animaux de race Piétrain, par rapport aux Large White, en particulier au niveau du Gluteus medius.

L'examen des notes attribuées d'après l'aspect des jambons montre que le traitement a eu peu d'influence sur la qualité appréciée subjectivement chez les animaux de race Large White. Bien que l'on constate une légère amélioration chez les porcs Piétrain, ceux-ci conservent après l'injection de sulfate de magnésium une qualité inférieure.

\section{DISCUSSION}

Les taux de glycogène déterminés immédiatement après l'abattage sont, dans le muscle Longissimus dorsi des animaux témoins, légèrement inférieurs à ceux que rapporte ChaRPENTIER (I968) pour des porcs abattus sans traitement préalable. Dans le cas présent les animaux étaient soumis à un jeûne plus prolongé ( 24 heures au lieu de $\mathrm{I} 2$ heures). D'autre part le glycogène est, in vivo, susceptible de variations quantitatives importantes en fonction des conditions d'environnement qui n'ont pas été contrôlées au cours de ces deux études. Enfin il peut exister des différences raciales considérables (SAYRE et al., I963) ce qui rend difficile la comparaison des résultats avec ceux que rapportent d'autres auteurs.

I.es teneurs initiales en composés phosphorylés (ATP et PC) chez les animaux témoins sont comparables aux résultats obtenus par SAIR et al. (I970) sur des porcs abattus sans traitement préalable. Ces auteurs rapportent des taux d'ATP et PC respectivement de $2,5 \mu$ moles $/ g$ et I $\mu$ mole/g environ chez les porcs présentant une chute de $\mathrm{pH}$ très rapide (cas des Piétrain); pour les animaux à chute de $\mathrm{pH}$ post 
mortem plus lente (comme les Large White de notre étude), ces valeurs sont plus élevées, environ 3,6 d'ATP et 4,5 $\mu$ moles de PC par gramme de tissu. Ces quantités sont, par contre, inférieures, surtout en ce qui concerne la phosphocréatine, aux résultats obtenus par CharpentTER (I968). Cette différence est peut-être en partie imputable à la différence entre les méthodes d'estimation utilisées, puisque CHARPENTIER employait pour le dosage de la phosphocréatine la technique de ENNOR-ROSENBERG.

L'augmentation des taux initiaux de glycogène, d'ATP et de PC dans tous les groupes d'animaux traités est due à l'action curarisante du sulfate de magnésium : en effet les contractions musculaires violentes qui accompagnent la saignée chez les animaux témoins consomment de l'ATP, qui ne peut être régénéré, après la suppression de l'apport d'oxygène, que par dégradation du glycogène et de la phosphocréatine ; la suppression des contractions permet donc le maintien de taux plus élevés de ces composés dans le muscle au moment de la mort (BENDal, ig65). Pour la même raison l'accumulation d'acide lactique, produit final de la glycogénolyse, est beaucoup plus faible chez les animaux injectés que chez les témoins ; par la suite l'élévation du $\mathrm{pH}$ initial favorise chez les premiers le fonctionnement du réticulum sarcoplasmique, permettant un déroulement lent et progressif de la glycogénolyse (GREASER et al., Ig69). Ce ralentissement de la glycogénolyse pourrait être dû. également à l'effet inhibiteur des ions $\mathrm{Mg}^{++}$sur l'activité ATPasique activée par le calcium des myofibrilles et sur le relargage du calcium par le réticulum sarcoplasmique (EBASHI et al., I969). Il faudrait toutefois que ces ions pénètrent dans la fibre musculaire rapidement et en quantité suffisante pour modifier leur concentration intracellulaire, ce qui n'est pas démontré.

Il est à remarquer que l'efficacité de l'injection de sulfate de magnésium paraît étroitement liée au type métabolique du muscle. Elle est beaucoup plus nette, chez les deux races, dans le Rectus abdominis que dans le Longissimus dorsi. Cette constatation pourrait expliquer le fait que, après traitement, la chute de $\mathrm{pH}$ dans le Longissimus dorsi reste plus rapide chez les Piétrain que chez les Large White: (o,3I unités $\mathrm{pH} /$ heure chez les premiers, contre $0, \mathrm{I} 8$ unités $\mathrm{pH} /$ heure chez les seconds pendant les 3 premières heures succédant 1'abattage) : le Longissimus dorsi des Large White est plus riche en fibres rouges et possède plus de capillaires par unité de surface que celui des Piétrain (résultats non publiés). Il est possible que l'effet curarisant des ions magnésium soit plus prononcé dans les muscles rouges, mieux irrigués, par suite d'un apport supérieur de ces ions par la circulation sanguine et d'une meilleure répartition dans le tissu musculaire. En outre, les fibres rouges et les fibres blanches présentent, du point de vue de l'activité ATPasique myofibrillaire et du fonctionnement du réticulum sarcoplasmique, des divergences importantes : on peut penser que le magnésium agit, à ce niveau, avec une intensité différente selon le type métabolique de la fibre, dans la mesure bien entendu où sa concentration intracellulaire est modifiée.

Dans le Longissimus dorsi les modifications de la glycogénolyse post mortem consécutives à l'injection de sulfate de magnésium sont beaucoup moins spectaculaires que ne l'indiquent SAIR et al. (I970) : la cause en est sans doute que ces auteurs maintenaient leurs animaux sous perfusion constante d'ions $\mathrm{Mg}^{++}$pendant les vingt minutes précédant l'abattage, et obtenaient ainsi une curarisation plus complète et peut-être des modifications de la concentration intracellulaire en magnésium plus prononcées. 
L'amélioration du pouvoir de rétention d'eau de la viande, chez les animaux traités des deux races, est une conséquence directe du ralentissement de la chute de $\mathrm{pH}$ post mortem. Les notes subjectives traduisent assez mal cette amélioration de la qualité, surtout chez les Large Whito : en fait, elles résultent d'une appréciation globale de la qualité, tenant compte de la couleur et de la tenue des muscles du jambon ; ces caractéristiques sont moins nettement modifiées que le pouvoir de rétention d'eau par le traitement. Il n'est pas surprenant que le pouvoir de rétention d'eau des différents muscles étudiés soit dans tous les cas inférieur chez les porcs Piétrain, puisque même après l'injection la glycogénolyse reste plus rapide dans le Longissimus dorsi de ces animaux que dans celui des Large White; les muscles du jambon sur lesquels cette caractéristique a été mesurée, le Gluteus medius et le Biceps femoris, sont comme le Longissimus doris riches en fibres blanches et leur évolution post mortem est sans doute comparable.

\title{
CONCLUSION
}

Chez le Porc, l'injection intraveineuse de sulfate de magnésium immédiatement avant l'abattage induit un ralentissement de la glycogénolyse post mortem, principalement semble-t-il grâce à la suppression de la transmission des stimulations nerveuses au muscle. Les résultats de ce traitement diffèrent cependant selon la race et le muscle considéré, ce qui nous conduit à penser que l'effet des ions $\mathrm{Mg}^{++}$administrés ante mortem est lié à d'autres facteurs physiologiques tels que l'irrigation sanguine et le type métabolique des fibres musculaires. Bien que l'on constate une amélioration du pouvoir de rétention d'eau de la viande, la suppression des stimulations nerveuses au moment de l'abattage ne peut suffire à assurer dans tous les cas l'obtention d'une viande de qualité satisfaisante, surtout dans la race Piétrain ; ceci d'autant plus que les muscles blancs, qui constituent l'essentiel des morceaux chers de la carcasse, sont les moins affectés par un tel traitement.

Reçu pour publication en avril 1971 .

\section{SUMMARY}

\author{
POST MORTEM GLYCOGENOL,YSIS IN THE PIG MUSCLE AS \\ INFLUENCEI BY AN INJECTION OF MAGNESIUM SULPHA'TE
}

The effect of an intravenous injection of magnesium sulphate (o. $5 \mathrm{~g} / \mathrm{kg}$ live weight) immediately before slaughtering upon the post mortem muscle glycogenolysis was studied in 7 Large White pigs and 5 Piétrain pigs. These animals were compared with an equal number of controls from each breed. By means of this treatment, nervous stimulations to the muscle during slaughtering can be suppressed. Glycogenolysis was observed in the Longissimus dorsi and Rectus abdominis muscles owing to $\mathrm{pH}$ measurement and determination of glycogene, ATP, phosphocreatine and lactic acid during the three hours post mortem.

The treatment resulted in a slackening of the $\mathrm{pH}$ fall and of the post mortem glycogenolysis in the two muscles, but mainly in the Rectus abdominis. The effect of the treatment upon the Longissimus dorsi was higher in the Large. White pigs than in the Pietrain pigs. The relationships between the effect of magnesium sulphate and certain physiological characteristics of the muscle, such as blood irrigation and the metabolic type of the fibers, are discussed. 
The treatment gives rise to an improvement of the meat quality observed 24 hours post mortem, and particularly of the water-binding capacity. Nevertheless, this effect is not sufficient enough to obtain meat of satisfactory quality, especially with regard to the Piétrain breed.

\section{RÉFÉRENCES BIBLIOGRAPHIQUES}

Beecher G. R., Cassens R. G., Hoekstra W. C., Briskey I.. J., i965. Red and White fibers content and associated post mortem properties of seven porcine muscles. J. Food Sci., 30, 969*976.

BENDALI J. R., r965. The effect of pretreatment of pigs with curare on the post mortem rate of pH fall and onset of the rigor mortis in the musculature. J. Sci. Food Astr., 17, 333-337.

Bergmeyer M. U., I965. Methods of enzymatic analysis. Academic Press, N. Y., London, and edition I064 p.

Briskey b. J., Wismer-Pedersen J., ig6I. I3iochemistry of pork muscle structure. I. Rate of anaerobic glycolysis and temperature change versus the apparent structure of muscle tissue. J. Food Sci., 26, $297-305$.

Bugard P., Henry M., Joubert L., ig62. Maladies de civilisation et dirigisme biologique. Sélection, élevage, alimentation, 197 p. Masson et $\mathrm{C}^{\mathrm{ie}}$, éditeurs. Paris.

Charpentifr J., 1968. Glycogénolyse post montem du muscle Ion aissimus dorsi de porc. Ann. Zootech., 17, $429 \cdot 443$.

Ebashi S., Endo M., Ortsuki I., r969. Control of muscle contraction. Quart. Rev. Biophys., 2, $35 \mathrm{I}-384$.

Greaser M. L., Cassens R. G., Hoekstra W. C., Briskey l. J., ig69. The effect of pH-temperature treatment on the calcium accumulating ability of purificd sarcoplasmic reticulum. J. Food Sci,. 34, $633-636$.

Huguett A. S., Nixon D. A., 1957. Enzymatic determination of blood glucose. Biochem. J., 66, I 2 p.

Lister I), I968. Sone aspects of the physiology of pale, soft and exsudative muscle in Recent points of view on the condition and meat quality of pigs for slaughter. p. I23-I32, edited by W. Sybesma, P. G. van der Wal and P. Walstra. Research Institute for Animal Husbandry-Zeist. The Netherlands.

Lister D., Sair R. A., Will J. A., Schimidt G. R., Cassens R. G., Holekstra W. C., Briskey E. J., I970. Metabolism of striated muscle of stress-susceptible pigs breathing oxygen or nitrogen. Amer. J. Physiol., 218, 102-ro7.

Ludvigsen J., I957. On the hormonal regulation of vasomotor reactions during exercise with special reference to the action of adrenal cortiral steroids. Acta Endocrin., 26, 406-4r6.

Sair R. A., I.ister D., Moody W. G.. Cassens R. G., Hoekstra W. C., Briskey E. J., i970. Action of curare and magnesium on striated muscle of stress-susceptible pigs. Amer. J. Physiol., 218, IC8-II4.

SAYre R. N., BRiskey E. J., Hoekstra W. C.., 1963. Porcine muscle glycogen structure and its association with other muscle properties. Proc. Expl. Biol. Med., 112, 223-225. 